\title{
Metastasis of hypopharyngeal squamous cell carcinoma to the kidney-a case report
}

\begin{abstract}
Background: Metastases to the kidneys are a relatively rare entity as the $5^{\text {th }}$ most common site for metastatic spread of solid tumors following the lungs, liver, bones and adrenals. The most common location of a primary tumor to metastasize to the kidney is lung, followed by breast, gastro-intestinal tract and head and neck cancers. To our knowledge this is the first reported case of a head and neck squamous cell carcinoma that metastasized to the kidney for which the patient underwent nephrectomy and retroperitoneal lymph node dissection.

Case report: A 66year old male with a past medical history of T3c N2b M0 hypopharyngeal squamous cell carcinoma presented to Urology clinic for consultation regarding a left renal mass. The patient underwent a laparoscopic left nephrectomy and retroperitoneal lymph node dissection. Pathology returned a $10 \mathrm{~cm}$ ill-defined mass located in left kidney and a $4.5 \mathrm{~cm}$ mass in the resected lymph nodes with extra nodal extension. Tumor markers and gene mutations were consistent with the patient's history of head and neck squamous cell carcinoma.

Discussion: Metastatic disease to the kidneys is a rare occurrence that can be challenging to manage, with head and neck serving as a rare source for the primary tumor. The laparoscopic nephrectomy was performed with curative intent as the patient had no evidence of recurrence elsewhere at the time of surgery, with the assumption that the mass was a primary RCC. This case exemplifies the role for percutaneous renal biopsy in patients with a history of primary malignancies in other organ systems.
\end{abstract}

Keywords: nephrectomy, renal mass, hypopharyngeal squamous cell carcinoma
Volume 4 Issue 6 - 2016

\author{
Michael Patton W,' Abdul-Muhsin Haidar,' \\ Matthaeus Jaime $\mathrm{L},{ }^{2} \mathrm{Ho}$ Thai $\mathrm{H},{ }^{3}$ Stanton \\ Melissa L, ${ }^{4}$ Castle Erik P,' Lott David G ${ }^{2}$ \\ 'Department of Urology, Mayo Clinic Hospital, USA \\ ${ }^{2}$ Department of Otorhinolaryngology-Head and Neck Surgery, \\ Mayo Clinic Hospital, USA \\ ${ }^{3}$ Department of Hematology and Oncology, Mayo Clinic \\ Hospital, USA \\ ${ }^{4}$ Department of Laboratory Medicine and Pathology, Mayo \\ Clinic Hospital, USA
}

Correspondence: Michael Patton, Department of Urology, Mayo Clinic Hospital, 5779 E Mayo Blvd, Phoenix, AZ 85054, USA,Tel (520) 977-I358, Email patton.michael@mayo.edu

Received: October 09, 2016 | Published: November 04, 2016
Abbrevations: PET, positron emission tomography; FDG, flu deoxy glucose; VHL, von hippel lindau; PBRM1, protein polybromo-1; BAP1, brca1 associated protein 1; SETD2, set domain containing 2; ERCC1, dna excision repair protein; HPV, human papilloma virus

\section{Introduction}

Metastases to the kidneys are a relatively rare entity and can be challenging to manage, requiring a multidisciplinary approach. The kidney is the 5th most common site for metastases of solid tumors following the lungs, liver, bones and adrenal glands. ${ }^{1}$ The most common location of a primary tumor to metastasize to the kidney is lung, followed by breast, gastro-intestinal tract and head and neck cancers. ${ }^{1}$ Most of the data regarding the incidence of metastatic disease to the kidneys come from autopsy studies dating back to the 1950 's, with incidences ranging from $7.2 \%$ to $12.6 \%$. $^{2,3}$ To our knowledge this is the first reported case of a head and neck squamous cell carcinoma that metastasized to the kidney for which the patient underwent nephrectomy and retroperitoneal lymph node dissection.

\section{Case report}

A 66-year-old male with a past medical history significant for hypopharyngeal squamous cell carcinoma presented for consultation for evidence of a renal mass on CT PET imaging, performed for surveillance of his head and neck cancer, two years after resection of his primary tumor. The patient underwent neo-adjuvant chemo radiation with cisplatin for his original tumor and subsequently underwent excision of the hypopharyngeal mass. Pathologic staging from this operation returned T3c, N2b, M0. ${ }^{4}$ The patient quickly developed a local recurrence and underwent a total laryngopharyngectomy with left and right neck dissections. Pathology revealed a hypopharyngeal, poorly differentiated grade 3 squamous cell carcinoma measuring $2.6 \mathrm{~cm} \times 2 \mathrm{~cm} \times 1.2 \mathrm{~cm}$ with perineural invasion, with no invasion of the thyroid, larynx or thymus but, microscopic involvement of superior margin. Tumor immunohistochemistry was positive for p63 and p53, and negative for $\mathrm{p} 16$. On right neck dissection 0 out of 9 lymph nodes returned positive and on left neck dissection 0 out of 21 lymph nodes were positive for an overall pathologic staging of $\mathrm{T} 2$, N0, M0. ${ }^{4} \mathrm{~A}$ year and a half later the patient underwent surveillance PET imaging which revealed FDG avidity involving a left renal mass and adjacent para-aortic lymph node (Figures 1-4). At the time, this was felt to be a primary renal mass, consistent with renal cell carcinoma. The patient then underwent a left laparoscopic radical nephrectomy and retroperitoneal lymph node dissection with curative intent.

The upper pole of the kidney was initially mobilized, followed by the inferior pole. As the kidney was lifted anterolaterally, the suspicious node was easily identified on the anterolateral surface of the aorta and the renal mass was identified involving the majority of the posterior portion of the kidney. Lymphadenectomy included pre-aortic, para-aortic, and down to and including the left common iliac lymph node packet. Operative time was 90 minutes with $50 \mathrm{~mL}$ of blood loss. Pathology from this surgery returned a $10 \mathrm{~cm}$ ill-defined mass located in left kidney with involvement of renal sinus fat and perinephric adipose tissues with negative margins. The lymph node revealed a $4.5 \mathrm{~cm}$ mass with extra nodal extension (Figure $5 \& 6$ ). Clinical Laboratory Improvement Amendments (CLIA)-certified mutation profiling was negative for common renal cell carcinoma gene mutations including VHL, PBRM1, BAP1, or SETD2. Loss of function mutations, consistent with a head and neck primary tumor, were identified in p53 and ERCC1. HPV (family 16) in situ 
hybridization was negative for types 16, 18, 31, 33, and 51 (Figure 7). Given these mutations and the morphology of the tumor, they were deemed consistent with metastasis of the patient's hypopharyngeal squamous cell carcinoma. Unfortunately, subsequent imaging three months after nephrectomy revealed recurrent disease in the head and neck as well as probable mesenteric metastases. The patient has been started on single agent cetuximab therapy with evidence of response to therapy on most recent CT imaging 5months post nephrectomy.

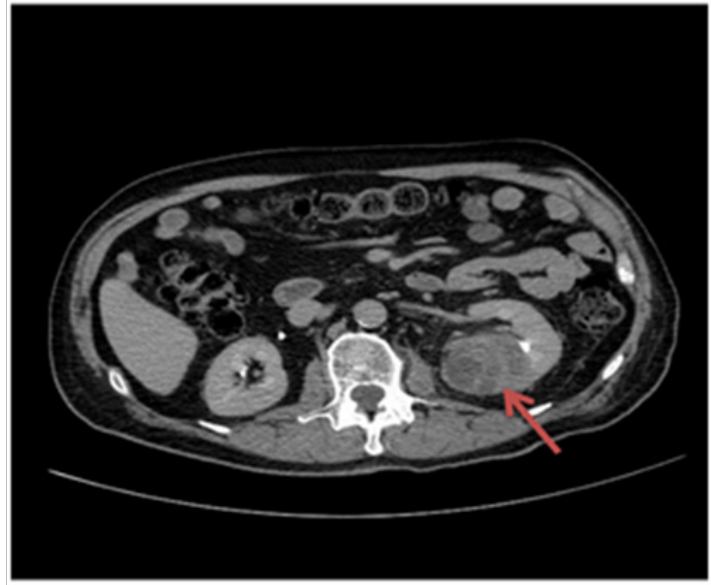

Figure I Axial image of renal mass.

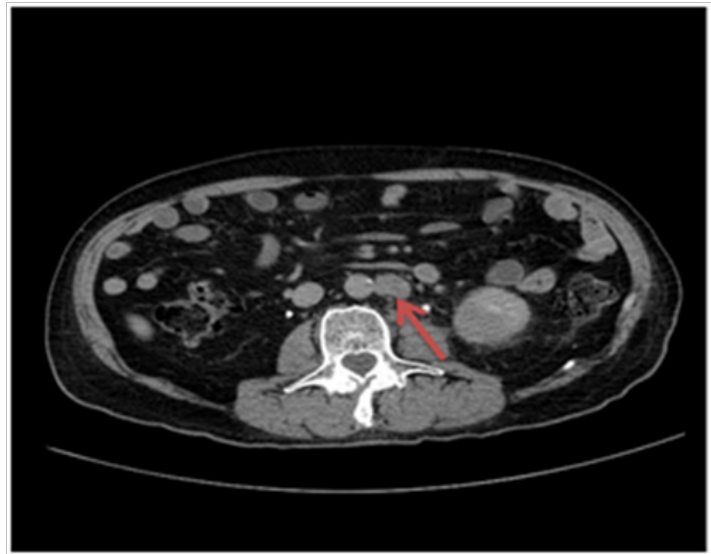

Figure 2 Axial image of para-aortic lymph node.

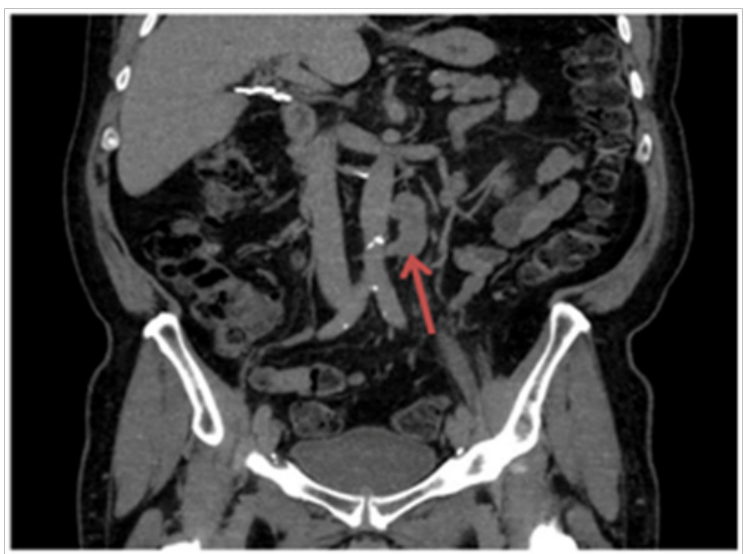

Figure 3 Coronal image of para-aortic lymph node.

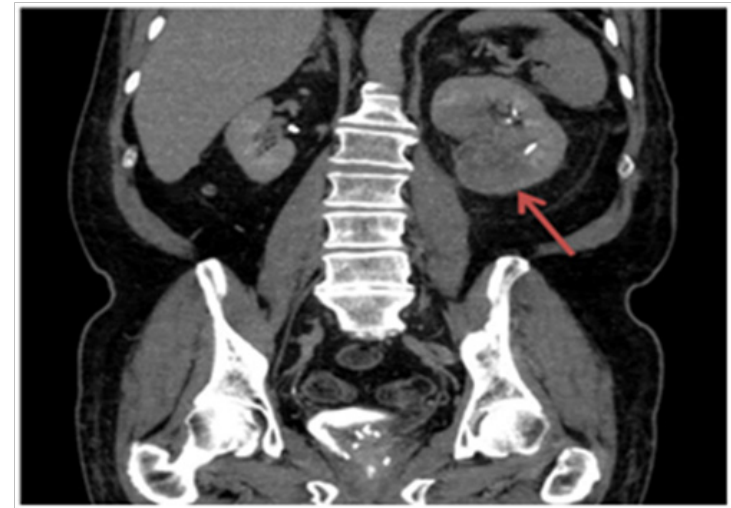

Figure 4 Coronal CT image of renal mass.

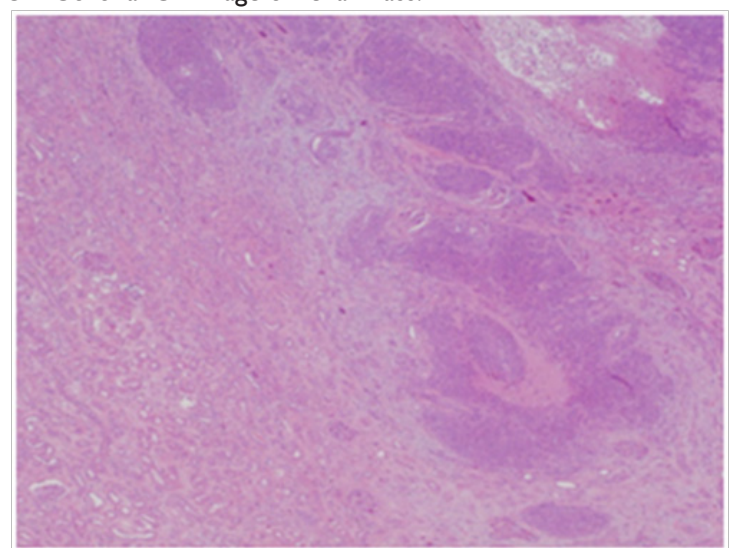

Figure 5 Hematoxylin and eosin stain of tumor at $4 x$ magnification.

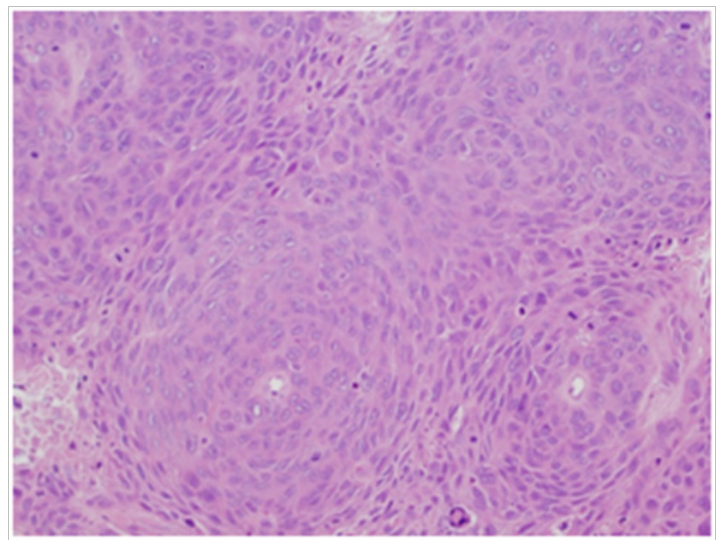

Figure 6 Hematoxylin and eosin stain of tumor at 20x magnification.

\section{Discussion}

Metastatic disease to the kidneys is a rare occurrence that can be challenging to manage, with head and neck serving as a rare source for the primary tumor. The majority of studies of head and neck cancers report clinical rates of distant metastases of approximately $15-25 \%,{ }^{5}$ with squamous cell carcinoma being the predominant tumor type. In these studies, stage IV tumors with advanced nodal disease and tumors of the hypopharynx had the highest incidence of distant metastases, most commonly to the lung, bone or liver. ${ }^{5}$ To our knowledge, no study exists looking specifically at renal metastases from head and neck malignancies. 


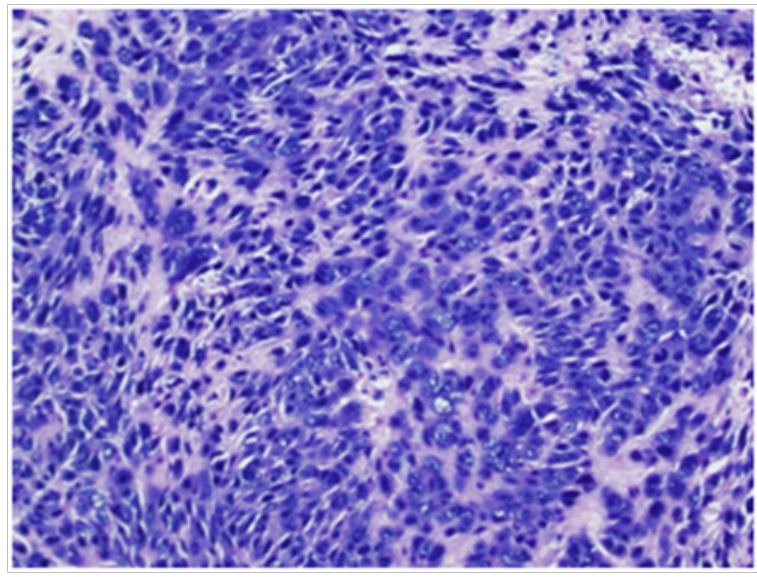

Figure 7 In Situ Hybridization (ISH) studies for Human Papilloma Virus (HPV) DNA.

Renal metastases of a distant primary represent only $3 \%$ of all surgically resected renal masses. A retrospective review of 43 surgical pathology cases of renal metastases found only $4(9.3 \%)$ of the tumors originated in the head and neck, three of which were thyroid primaries and one was a carcinoma ex-pleomorphic adenoma from salivary gland. ${ }^{3}$ In another retrospective review of 151 patients with metastatic disease to the kidneys, $6 \%$ of the primary tumors originated in the head and neck, while $80.8 \%$ originated in the lungs. ${ }^{6}$ Of these tumors, $77.5 \%$ were solitary and only $32 \%$ of patients underwent a nephrectomy, including eight out of nine metastatic head and neck cancer patients. This study also found that over half of patients did not have any symptoms from renal metastases, with the most common symptom being flank pain in up to $30 \%$ of individuals. In terms of survival, the study reported an overall median survival after diagnosis of renal metastases of approximately 14 months. Patients who underwent surgery (partial or radical nephrectomy) did show a significant survival benefit compared to those who did not (median 2.24 years versus 0.72 years), though a number of factors contributed to this. Surgery was done with curative intent in 21 patients (44.7\%), for a suspected primary tumor in 11 patients $(23.4 \%)$, for chemotherapy failure/palliation in 10 patients $(21.3 \%)$, and for non-functioning symptomatic kidney in five patients $(10.6 \%){ }^{6}$

Our patient's imaging was consistent with a renal cell carcinoma with some concern for possible metastatic disease, but, as he had no evidence of other recurrence from his hypopharyngeal squamous cell carcinoma, a nephrectomy with lymph node dissection was performed with curative intent. There has been increasing interest in percutaneous renal biopsy for indications including tissue diagnosis of radiologic indeterminate renal masses to avoid resecting benign lesions and for selecting appropriate medical and surgical treatment strategy in the setting of metastatic renal cell carcinoma. ${ }^{7}$ One established role of renal biopsy is the presence of a solid renal mass and a known extrarenal primary malignancy. ${ }^{8}$ In this case, a renal biopsy may have been indicated, though in the absence of other systemic disease at the time, a nephrectomy with lymphadenectomy may have still been indicated prior to chemotherapy. The patient had close follow-up with oncology and unfortunately, as is often the case with advanced head and neck cancer, the patient experienced a local recurrence in the neck as well as mesenteric metastases on imaging three months after nephrectomy. The patient was started on single agent cetuximab therapy at that time and recent imaging, approximately 6months status post nephrectomy, shows response to therapy. This case illustrates the importance of a multimodal approach to the management of aggressive cancers with the propensity to metastasize and an instance where percutaneous biopsy of a renal mass may be indicated.

\section{Acknowledgments}

None.

\section{Conflicts of interest}

Author declares there are no conflicts of interest.

\section{Funding}

None.

\section{References}

1. Wu AJ, Mehra R, Hafez K, et al. Metastases to the kidney: a clinicopathological study of 43 cases with an emphasis on deceptive features. Histopathology. 2015;66(4):587-597.

2. Bracken RB, Chica G, Johnson DE, et al. Secondary renal neoplasms: an autopsy study. South med J. 1979;72(7):806-807.

3. Abrams HL, Spiro R, Goldstein N. Metastases in carcinoma, analysis of 1000 autopsied cases. Cancer. 1950;3(1):74-85.

4. National Comprehensive Cancer Network. NCCN Clinical Practice Guidelines in Oncology: Head and Neck Cancers. National Comprehensive Cancer Network, USA. 2015.

5. Ferlito A, Shaha AR, Silver CE, et al. Incidence and sites of distant metastases from head and neck cancer. ORL J Otorhinolaryngol Relat Spec. 2001;63(4):202-207.

6. Zhou C, Urbauer DL, Fellman BM, et al. Metastases to the kidney: a comprehensive analysis of 151 patients from a tertiary referral centre. BJU Int. 2015;117(5):775-782.

7. Ljungberg B, Bensalah K, Canfield S, et al. EAU guidelines on renal cell carcinoma: 2014 update. Eur Urol. 2015;67(5):913-924.

8. Caoili EM, Davenport MS. Role of percutaneous needle biopsy for renal masses. Semin Intervent Radiol. 2014;31(1):20-26. 